\title{
Valorización económica ecológica de árboles de Bertholletia excelsa por el método producción-daños en Madre de Dios (Perú)
}

\author{
Ecological economic valuation of trees of Bertholletia excelsa \\ by the production-damage method in Madre de Dios (Peru)
}

\author{
Emer R. Rosales ${ }^{1, *}$
}

Recibido: 20 julio 2019 | Aceptado: 14 junio 2020 | Publicado en línea: 01 agosto 2020 Citación: Rosales, ER. 2020. Valorización económica ecológica de árboles de Bertholletia excelsa por el método producción-daños en Madre de Dios (Perú). Revista Forestal del Perú 35(1): 42-53. DOI: http://dx.doi.org/10.21704/rfp.v35i1.1476

\begin{abstract}
Resumen
La presente investigación, realizada en el departamento de Madre de Dios, tuvo por objetivo la valorización económica ecológica de los árboles de Bertholletia excelsa Bonpl. (castaña amazónica) en cuatro concesiones forestales. La metodología aplicada fue la valoración objetiva por el método de producción-daños. Este método consideró: la valorización de la producción de frutos, a través del método de producción y la valorización por daño como árbol o valor per se, aplicando el método ICONA, la suma de ambos valores genera el valor económico ecológico. Para la valorización directa, con base en la producción de la castaña en cáscara por árbol de $B$. excelsa se reportan valores promedio de $\mathrm{S} / .67,42 /$ árbol y $\mathrm{S} / .1,78 / \mathrm{kg}$ de producto. El valor per se promedio por árbol en pie fue S/. 3 941,44/árbol. El valor económico ecológico promedio por hectárea de concesión resultó S/. 2 146,78/ha. Se concluyó que el valor de producción de la castaña en cáscara, el valor per se por árbol en pie y el valor económico ecológico del árbol de B. excelsa de las concesiones ubicadas en Tambopata, Las Piedras, Laberinto y Tahuamanu son diferentes y ostentan un coeficiente de variación alto, además las concesiones presentan una correlación positiva y significativa entre valoración económica ecológica y número de árboles.
\end{abstract}

Palabras clave: valor de producción, costo unitario, valor existencial, Tambopata, Tahuamanu

\footnotetext{
${ }^{1}$ Servicio Nacional Forestal y de Fauna Silvestre (SERFOR CAF), Jr. Jorge Chávez 1160, Puerto Maldonado, Madre de Dios, Perú.

* Autor de Correspondencia: errs1973@gmail.com
} 


\begin{abstract}
The present investigation, carried out in the department of Madre de Dios, aimed at the ecological economic valuation of the Bertholletia excelsa Bonpl. trees (Amazon chestnut) in four forest concessions. The applied methodology was the objective assessment by the production-damage method. This method considered: the valuation of fruit production, through the production method and the valuation for damage as a tree or value per se, applying the ICONA method, the sum of both values generates the ecological economic value. For direct recovery, based on the production of the chestnut in shell per tree of B. excelsa, average values of $S / .67 .42 /$ tree and $S / .1 .78 / \mathrm{kg}$ of product are reported. The average per se value per standing tree was S/. $3941.44 /$ tree. The average ecological economic value per hectare of concession was S/. 2 146.78/ha. It was concluded that the production value of the chestnut in shell, the value per se per standing tree and the ecological economic value of the B. excelsa tree from the concessions located in Tambopata, Las Piedras, Laberinto and Tahuamanu are different and boast a high coefficient of variation, in addition the concessions present a positive and significant correlation between ecological economic valuation and number of trees.
\end{abstract}

Key words: production value, unit cost, existential value, Tambopata, Tahuamanu

\section{Introducción}

La castaña (Bertholletia excelsa Bonpl.) es una especie originaria del Sur-Este amazónico, distribuida en la Cuenca Amazónica, en Bolivia, Brasil, Colombia, Perú, Venezuela, Guyana y Surinam. En el Perú se encuentra en la Selva Baja, en estado natural, cultivado y en producción en el departamento de Madre de Dios, y sólo cultivado en el departamento de Loreto y Ucayali. Sus requerimientos ambientales adaptativos son: Precipitación pluvial promedio anual de 1400-2800 $\mathrm{mm}$; temperatura promedio anual de $24-27^{\circ} \mathrm{C}$; humedad relativa media anual de 79-91 \%; y la altitud de 0-500 $\mathrm{m}$ (Flores 1997). No le afectan regímenes rústicos, con lluvias mensuales inferiores a $100 \mathrm{~mm}$ durante 4 a 5 meses. Prospera en terrenos no inundables, en oxisoles y ultisoles ricos en materia orgánica, arcillosos o arcilloso-arenosos, ácidos, pobres en nutrientes, con buen drenaje y no tolera saturación hídrica (Rosales 2012a). El árbol de la castaña es muy alto, pudiendo medir de 30 a $50 \mathrm{~m}$ de altura (Flores 1997). Asimismo, Brienen (2005), en su reporte sobre anillos de árboles en los trópicos registró, mediante dataciones de radios carbónicas, una edad de 427 años para árboles de $180 \mathrm{~cm}$ de diámetro y de $440 \pm 60$ años para árboles de $233 \mathrm{~cm}$ de diámetro.
El fruto de la castaña es una cápsula de forma globosa o esférica, presenta una corteza dura y leñosa, mide de 9 a $15 \mathrm{~cm}$ de diámetro y pesa entre 0,5 y $1,5 \mathrm{~kg}$. Un árbol maduro puede dar entre 200 y 400 frutos. Dentro del fruto hay entre 10 y 25 semillas, de 3 a $5 \mathrm{~cm}$ de largo y 4 a 10 gramos de peso. Las semillas de castaña tienen una cubierta rugosa, dura y leñosa, y en su interior una almendra de color blanquecino envuelta en una epidermis marrón. Aunque la producción de un árbol de castaña es muy variable, se estima que puede dar de 100 a 120 kilos de semillas. En el bosque natural, la fructificación se inicia entre los 12 y 16 años, con buen manejo en espacios abiertos, puede reducirse a 8 años. Árboles injertados a los 12 meses producen $25 \mathrm{~kg}$ de castaña, cantidad superior a la producción de árboles nativos que en promedio producen de $5-14 \mathrm{~kg} /$ planta. La cosecha es manual, los frutos maduros caen del árbol y son recolectados del suelo, durante la estación lluviosa, generalmente por la mañana. Por la tarde, el fruto es abierto con un machete. Las semillas extraídas se lavan inmediatamente, después de ser secadas por pocas horas son almacenadas bajo techo. El secado posterior, se realiza bajo el sol por algunos días y su almacenamiento debe ser en un ambiente seco, lo cual favorece la calidad de la semilla para su procesamiento, lo que mejora el precio del producto (Flores 1997). 
Valorización económica ecológica de árboles de Bertholletia excelsa por el método producción-daños en Madre de Dios (Perú)

El aprovechamiento de la castaña en Madre de Dios comienza con la recolección del fruto y finaliza con la colocación del producto y sus distintos subproductos en el mercado internacional, mayoritariamente europeo y norteamericano. Este proceso de aprovechamiento del producto pasa por las distintas etapas y diversos actores de la cadena productiva, que conforman los siguientes eslabones: recolección y almacenamiento post cosecha, procesamiento artesanal y transporte a Puerto Maldonado, procesamiento industrial y comercialización de esta nuez amazónica. Sin embargo, la evaluación de la estructura de costos es compleja debido a las diferentes realidades que enfrentan los castañeros para la recolección y procesamiento del producto. Siendo la estructura de costos realizado en el año 2005 de S/. 1,07 y 1,55/kg por la Cooperativa de Recolectores Orgánicos de la Nuez Amazónica del Perú (RONAP) de Madre de Dios (PROMAMAZONIA 2019). Los costos generados a lo largo de la cadena de la castaña amazónica, realizada en el marco del festival de la castaña en Madre de Dios por empresas de Bolivia para el año 2010 representa para el rubro de recolección de castaña amazónica en cáscara o producto final un valor de S/. 2,07/kg (Gamarra 2014). Asimismo, un estudio realizado en Brasil reportó que los costos por recolección de castaña fueron de R $7,15 /$ lata, lo cual en soles seria hoy en día S/ 1,49 / kg aproximadamente (de Sá et al. 2004).

Por otro lado, un trabajo en Madre de Dios con los métodos semejantes al presente estudio concluye que, el valor económico de un árbol de castaña fue S/. 516,82/árbol y específicamente por su valor de producción de frutos de castaña pelada S/. 94,42/árbol y por su valor per se S/. 422,40/árbol (Rosales 2012b).

La presente investigación debe servir como una guía para todas las personas naturales o jurídicas que cuentan con concesiones castañeras, y debe de ser del conocimiento de directores, colaboradores, proveedores, clientes, etc. que puedan estimar la valorización económica ecológica de la castaña con el fin de hacer costos de manejo por mejoras del castañal y resolver conflictos económicos por daños. Debido a ello, los objetivos de este trabajo fueron: determinar y comparar el valor económico ecológico del total de árboles de Bertholletia excelsa en cuatro concesiones forestales, estimar el valor de su producción y estimar su valor existencial o per se.

\section{Materiales y Métodos}

\section{Ubicación política y geográfica}

Las áreas de estudio se encuentran ubicadas en el departamento de Madre de Dios, provincias de Tambopata y Tahuamanu, en los distritos de Tambopata sector Sabaluyoc (P1 469000E $8638040 N$ ), Las Piedras sector Loreto (P1 449300E $8682700 \mathrm{~N})$, Laberinto sector Lagarto (P1 404275E 8607000N) y Tahuamanu sector Alerta (P1 454779E 8695288N) (ver Figura 1).

\section{Valoración económica ecológica}

La metodología aplicada en esta investigación tuvo un enfoque de valoración objetiva por función producción-daños. Para ello, se utilizaron dos técnicas de valoración: la primera consistió en la valoración por el método de producción, lo cual se refiere al valor de uso con base en la producción de frutos de castaña. La segunda valoración como daño referido al valor de no uso, tipo existencial o per se por el aprovechamiento del legado ambiental, beneficios derivados por el conocimiento que se tiene del recurso, protección de hábitat, genes, conservación de la especie, ecosistemas únicos y prevención de hábitat de cambios irreversibles (Collazos 2009; Rosales 2012b; SERFOR CAF 2018). Seguidamente la suma de ambos valores definió el valor económico ecológico.

\section{A. Valoración de uso del árbol por su valor de producción (V1)}

Es la valorización de los árboles de la castaña en función de su producción de frutos impactada o dañada por la tala ilegal, agricultura migratoria y aprovechamiento irracional de otras especies comerciales maderables. Esta se calculó con la siguiente ecuación:

$$
\mathrm{V} 1=\mathrm{A} \times \mathrm{B} \times 1 / \mathrm{C}
$$




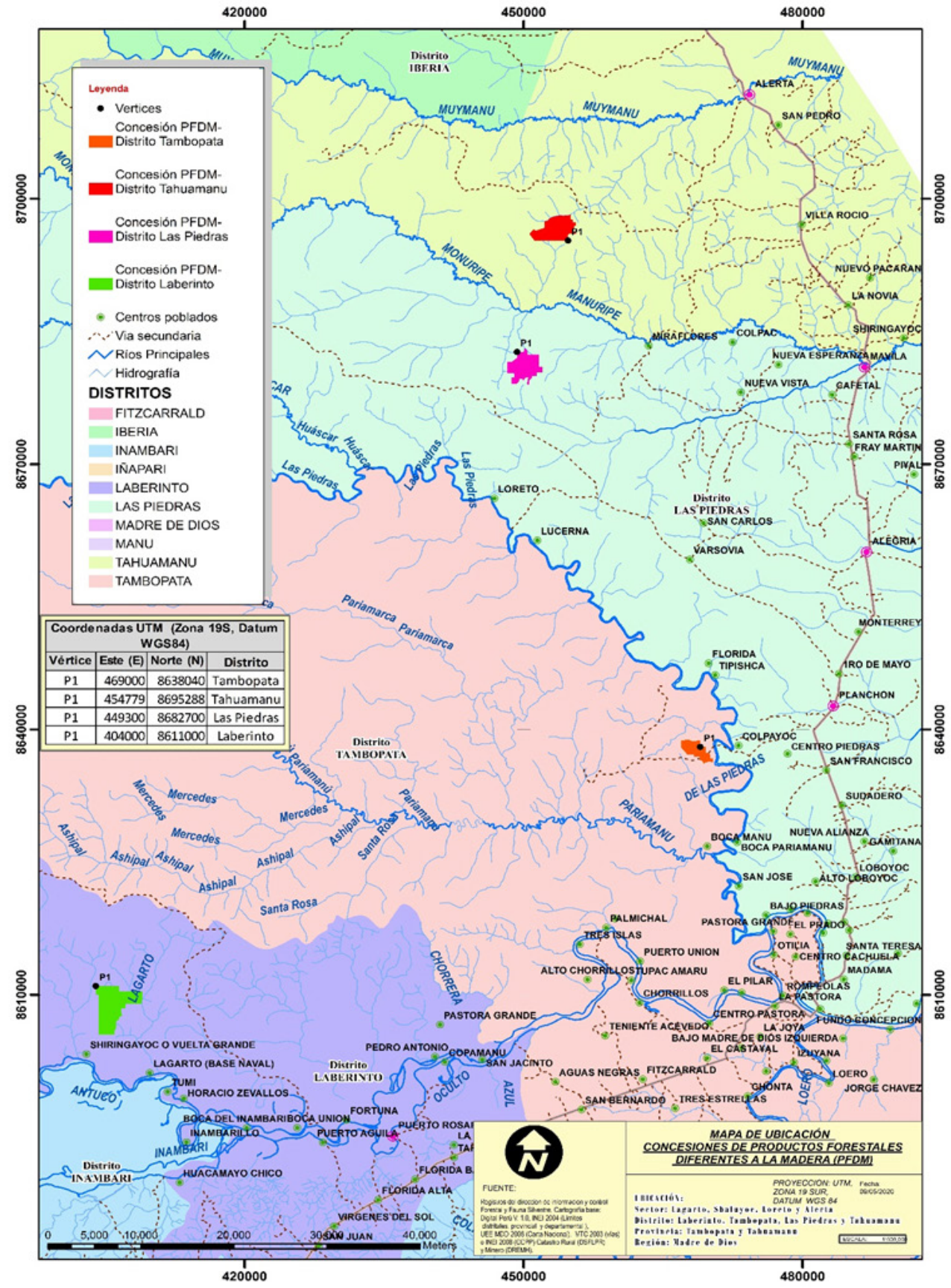

Figura 1. Mapa de ubicación política y geográfica de las concesiones. 
Valorización económica ecológica de árboles de Bertholletia excelsa por el método producción-daños en Madre de Dios (Perú)

Donde:

V1: Valor de uso del árbol por su valor productivo

A: Producción de frutos por árbol de castaña en condiciones normales.

B: Costo unitario de producción de frutos por kilogramo.

C: Cociente de riesgos y menor resistencia del arbolado (75\%).

El cálculo del valor productivo se realizó en base a los registros oficiales de producción de frutos por árbol en las cuatro concesiones visitadas y con base en los registros de campo del productor (DRFFS - MDD 2019, SERFOR CAF 2019a, SERFOR CAF 2019b).

\section{B. Valoración como daño de no uso del árbol} por su valor per se (V2)

Para función daño se tomó como referencias el método de valorización para árboles ornamentales según ICONA (1975). La valorización de los árboles de castaña en función del daño o deforestación causada por la tala ilegal, agricultura y aprovechamiento irracional de otras especies comerciales maderables (Rosales 2012). Se calculó con la siguiente ecuación:

$$
\mathrm{V} 2=\mathrm{A} \times \mathrm{B} \times \mathrm{C} \times \mathrm{D} \times \mathrm{E} \times \mathrm{F}
$$

Donde:

V2: Valor de no uso del árbol por su valor per se

A, B, C, D, E, F: Coeficientes del método ICONA

A: Clasificación según la especie.

B: Valor estético y funcional, y estado sanitario.

C: Situación.

D: Rareza.

E: Singularidad.

F: Relación edad y diámetro del árbol.

Los valores de los coeficientes utilizados para el cálculo del valor per se fueron hallados mediante encuestas. Para estas encuestas se usó un muestreo probabilístico y al azar, con la fina- lidad de que todos los sujetos a encuestar tuvieran la misma probabilidad de formar parte de la muestra. Se definió una población de 47 expertos forestales en $B$. ecxelsa en la ciudad de Puerto Maldonado, y para definir la muestra se usó la siguiente fórmula estadística:

$$
n=\frac{N \times P \times Q \times Z^{2}}{d^{2}(N-1)+Z^{2} \times P \times Q}
$$

Donde:

P: proporción de éxito $=0,95^{*}$

$\mathrm{q}=(1-\mathrm{p})=0,05$

$\mathrm{d}^{2}=$ Error muestral $=5 \%$

$\mathrm{Z}=1,96$ nivel de confianza al 95\%.

Resultando encuestar mínimamente 29 regentes forestales. Pero, por razones estratégicas del estudio y reducir el error estadístico se realizaron 36 encuestas a profesionales, los cuales fueron 29 regentes forestales y 7 consultores forestales académicos independientes de instituciones de Madre de Dios.

Finalmente, la estimación de la valorización económica ecológica del árbol de castaña resulta de la suma de la valorización económica del árbol por su valor de producción y su valor per se:

$$
\mathrm{Vt}=\mathrm{V} 1+\mathrm{V} 2
$$

Donde:

Vt $=$ Valoración económica ecológica del árbol de la castaña.

\section{Toma de datos}

El procedimiento para la toma de los datos y calcular los valores de V1 y V2, siguió tres etapas:

1) Etapa de pre campo.- Consistió en preparar los materiales, insumos e instrumentos para la etapa de campo como son los inventarios forestales y encuestas (SERFOR CAF 2018).

2) Etapa de campo.- Se realizó la actualización de los inventarios de los árboles de castaña, ello consistió en obtener datos dasométricos (diámetro, altura), morfométricos (estado fitosanitario, presencia de sogas 
entre otros), producción de frutos, ubicación georeferencial del árbol y costos del proceso de aprovechamiento de frutos de castaña. Que hoy con el nuevo reglamento de la Ley Forestal vigente sirven para actualizar las declaratorias de manejo (DEMA) de aprovechamiento de castaña. Durante esta etapa se realizaron encuestas técnicas a cada regente forestal y/o consultor forestal seleccionados al azar, las seis preguntas realizadas estuvieron enfocadas en la silvicultura, valor estético, situación, rareza, singularidad y relación edad y diámetro del árbol (SERFOR CAF 2019a y SERFOR CAF 2019b).

3) Etapa de gabinete.- Aquí se realizó un exhaustivo análisis de los datos obtenidos en el campo de la producción y costos del aprovechamiento de frutos de castaña. Asimismo, se solicitó información real y fidedigna de los ba- lances de extracción de castaña en cáscara de las cuatro concesiones en estudio del año 2007 al 2017, que al final promediaron la producción de los últimos 12 años incluido año 2018 (ver Figura 2). Seguidamente para el análisis y procesamiento de datos se utilizaron los programas SSPS versión 19 y Excel versión 10, para calcular la moda, promedios, desviación estándar, y coeficiente de variación, así como, cuadros y figuras estadísticas. Además de cálculos del coeficiente de correlación interpretados por Córdova (1997).

\section{Resultados}

En el Cuadro 1 se observa el área, número de árboles y el valor de producción de los frutos por árbol de castaña (V1) calculado por el producto de $\mathrm{A} \times \mathrm{B} \times 1 / \mathrm{C}$ en las cuatro concesiones ubicados en diferentes sectores y distritos del

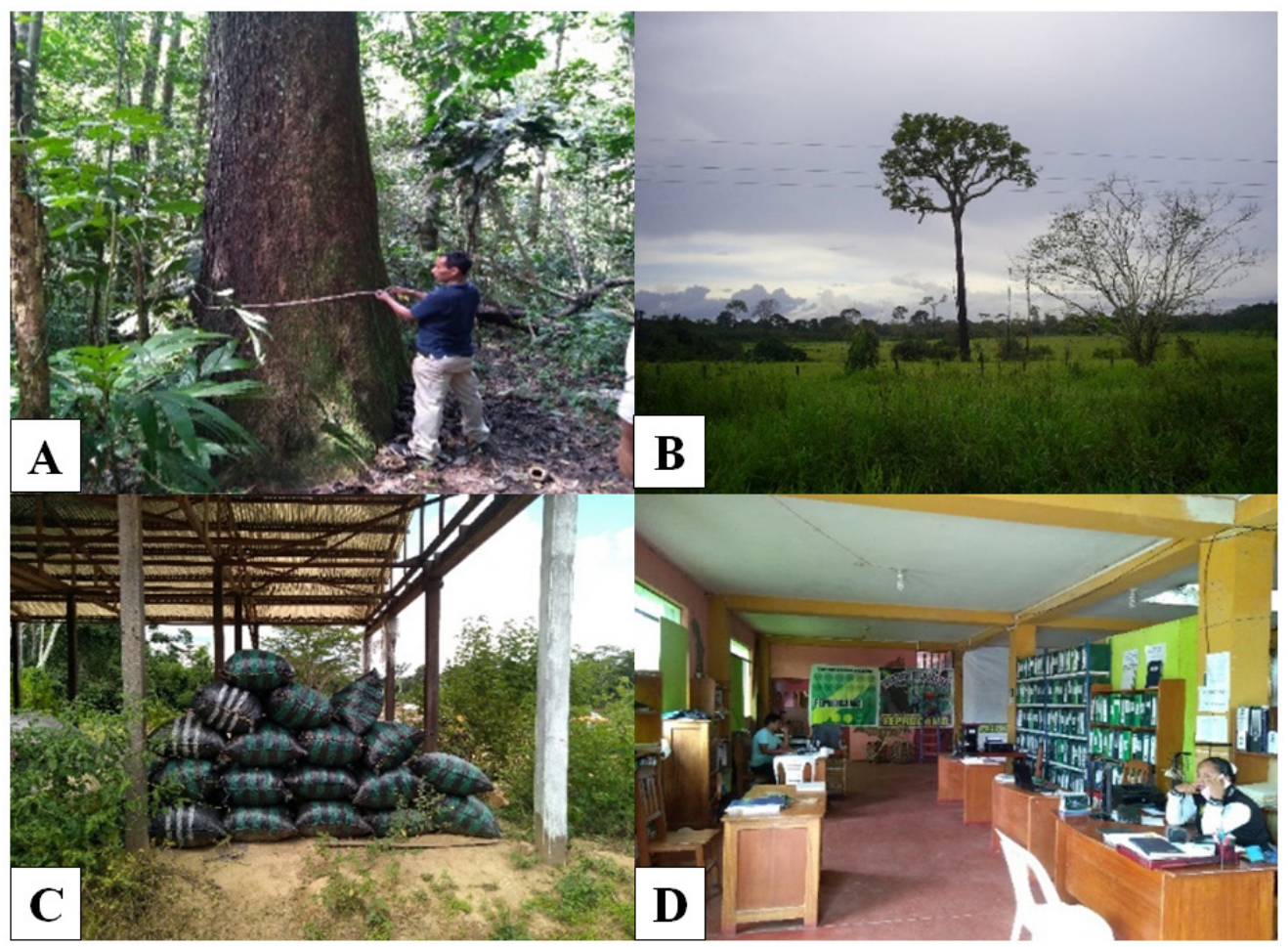

Figura 2. Etapas de toma de datos. A: actualización de inventarios. B: árbol de castaño en potrero. C: producción de castaña en barricas. D: encuesta a regentes y consultores forestales. 
Valorización económica ecológica de árboles de Bertholletia excelsa por el método producción-daños en Madre de Dios (Perú)

\begin{tabular}{|c|c|c|c|c|c|}
\hline \multirow[b]{2}{*}{ Rubros } & \multicolumn{4}{|c|}{ Concesiones de aprovechamiento de castaña } & \multirow{2}{*}{$\begin{array}{c}\text { Madre de } \\
\text { Dios } \\
\text { (promedios) }\end{array}$} \\
\hline & $\begin{array}{l}\text { Tambopata } \\
\text { (Sabaluyoc) }\end{array}$ & $\begin{array}{c}\text { Las Piedras } \\
\text { (Loreto) }\end{array}$ & $\begin{array}{l}\text { Laberinto } \\
\text { (Lagarto) }\end{array}$ & $\begin{array}{c}\text { Tahuamanu } \\
\text { (Alerta) }\end{array}$ & \\
\hline Área & 482,24 & 904,45 & 1592,65 & 975,79 & 988,78 \\
\hline Número de árboles & 169 & 872 & 410 & 667 & 530 \\
\hline $\begin{array}{l}\text { A: Producción frutos } \\
\text { (Kg/árbol) }\end{array}$ & 53,05 & 23,54 & 26,37 & 21,91 & 31,22 \\
\hline $\begin{array}{l}\text { B: Costo de producción por } \\
\text { kilogramo (soles } / \mathrm{Kg})\end{array}$ & 1,11 & 2,01 & 1,93 & 2,05 & 1,78 \\
\hline $\begin{array}{l}\text { C: Costo de riesgos y menor } \\
\text { resistencia del arbolado } \\
\text { (coeficiente) }\end{array}$ & 0,75 & 0,75 & 0,75 & 0,75 & 0,75 \\
\hline $\begin{array}{l}\text { V1: Valor producción por } \\
\text { árbol (soles) }\end{array}$ & 78,55 & 63,18 & 68,03 & 59,93 & 67,42 \\
\hline
\end{tabular}

Cuadro 1. Valor de producción de los frutos por árbol de Bertholletia excelsa Bonpl. (castaña) en las cuatro concesiones.

departamento de Madre de Dios. Donde la producción de frutos por árbol (A) producto del aprovechamiento de la cantidad de castaña por árbol, hasta el punto de venta fue para el sector de Sabaluyoc distrito de Tambopata S/. 53,05/árbol, Loreto distrito de Las Piedras S/. 23,54/árbol, Lagarto distrito de Laberinto S/. 26,37/árbol, Alerta distrito de Tahuamanu S/. 21,91/árbol y una media para el departamento de Madre de Dios de S/ 31,22/árbol. Seguidamente en ese orden se muestra que el costo unitario de producción por el aprovechamiento de los frutos de un kilogramo de castaña en cáscara (B) fue para el sector de Sabaluyoc S/. 1,11; Loreto S/. 2,01; Lagarto S/ 1,93; Alerta S/. 2,05 y una media para el departamento de Madre de Dios de S/. 1,78. Mientras que el coeficiente por riesgos $(\mathrm{C})$ para todos los sectores se contempló por fórmula 0,75 . Finalmente el valor de producción de frutos de castaña en cáscara por árbol para las concesiones de los mismos sectores y distritos fue S/. 78,55 (Tambopata), S/. 63,18 (Las Piedras), S/. 68,03 (Laberinto), S/ 59,93 (Tahuamanu) y un promedio calculado para la región de Madre de Dios de S/. 67,42.

En el Cuadro 2 se percibe el valor existencial o per se del árbol de castaña (V2) conformado por los siguientes seis índices: silvicultura de la especie (A), estética funcional y estado fitosanitario (B), situación poblacional (C), rareza $(D)$, singularidad de especie $(E)$, relación edad diámetro $(\mathrm{F})$. Este valor fue empleado en las cuatro concesiones, usando la moda y promediando los dos datos frecuentes de entre los cuatro distritos para determinar la media del departamento de Madre de Dios. El valor existencial evaluado como árbol en pie producto de $\mathrm{A} \times \mathrm{B} \times \mathrm{C} \times \mathrm{D} \times \mathrm{E} \times \mathrm{F}$, con criterios ecológicos in situ es diferente en las cuatro concesiones. Observando que un árbol en Sabaluyoc tuvo un valor de S/ 4 295,45; Loreto S/. 5 575,22; Lagarto S/. 7 706,42; Alerta S/. 2 500,00 y un valor estimado para la región de Madre de Dios S/. $3941,44$.

El Cuadro 3 muestra el área, número, densidad de árboles por hectárea y un resumen de valor de producción por árbol, valor existencial por árbol, valor económico ecológico por árbol, valor económico ecológico por total y valor económico ecológico por hectárea de las cuatro concesiones. Donde se percibe que el valor económico ecológico por árbol de la concesión por producción de frutos de castaña en cáscara y como árbol en pie con criterios ecológicos in situ son distintos en las cuatro concesiones, los cuales para el sector de Sabaluyoc distrito 


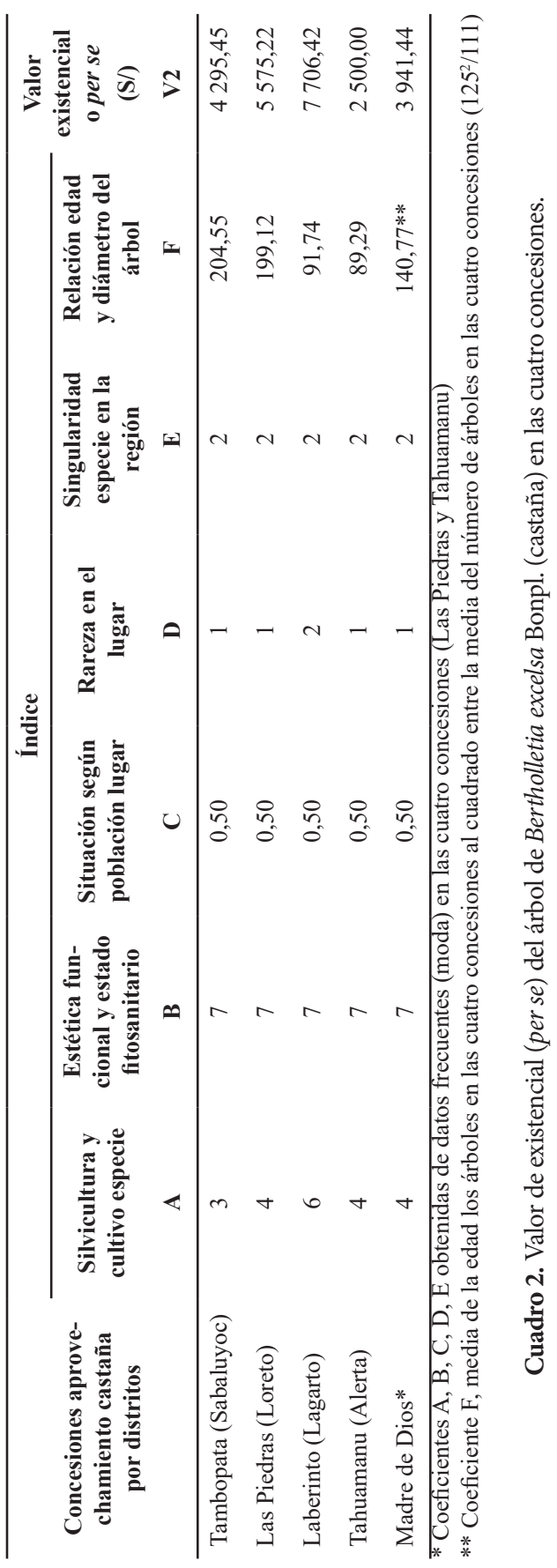

de Tambopata fue S/. 4 374,00; Loreto distrito de Las Piedras S/. 5 638,41; Lagarto distrito de Laberinto S/. 7 774,46 y Alerta distrito de Tahuamanu S/. 2 559,93. Seguidamente el valor para el total de los árboles o por concesión fue para el sector Sabaluyoc S/. 739 206,57; Loreto S/. 4916 690,22; Lagarto S/. 3187 526,63 y Alerta S/. 1707 474,80. Asimismo, se observa que el valor económico ecológico por hectárea de concesión en cada distrito fue de la siguiente manera: Tambopata S/. 1 532,86; Las Piedras S/ 5 436,11; Laberinto S/. 2 001,40 y Tahuamanu S/. 1749,84 .

En el Cuadro 4 se aprecia el número total de árboles y el valor económico ecológico para las cuatro concesiones, donde el valor económico ecológico de las concesiones tuvo una media aritmética para 529,50 árboles un valor de S/. 2637 724,56; una desviación estándar de S/. 1822 608,20 y un coeficiente de variación de $69,10 \%$ al 0,05 de confiabilidad, denotando alta variabilidad. Además, en la Figura 3 podemos ver que la relación entre el valor económico ecológico con el número total de árboles de castaña por concesión tuvo un coeficiente de correlación igual a 0,76 al 0,05 de confiabilidad que representa una correlación significativa para el estudio.

\section{Discusión}

La metodología utilizada para estimar los costos de aprovechamiento de producción de castaña fue la convencional, y sigue los pasos dentro de un plan de manejo forestal para productos diferentes de la madera (Rosales 2012b). Los resultados estimados en costos unitarios por kilogramo de castaña en cáscara aprovechada en Madre de Dios fueron de S/ $1,78 / \mathrm{kg}$, ligeramente menores en céntimos de sol a los estimados por Gamarra (2014) en Bolivia (S/. 2,07/kg); y ligeramente superiores a los calculados por la Cooperativa de Recolectores Orgánicos de la Nuez Amazónica del Perú (RONAP), reportados por PROMAMAZONIA (2019) para dos sistemas de aprovechamiento, en donde los costos fueron de S/. $1,07 / \mathrm{kg}$ y S/ 1,55/kg. De este mismo modo, nuestros resultados fueron ligeramente supe- 
Valorización económica ecológica de árboles de Bertholletia excelsa por el método producción-daños en Madre de Dios (Perú)

\begin{tabular}{|c|c|c|c|c|c|}
\hline \multirow[b]{2}{*}{ Rubros } & \multicolumn{4}{|c|}{ Concesiones de aprovechamiento de castaña } & \multirow[b]{2}{*}{$\begin{array}{l}\text { Madre de } \\
\text { Dios }\end{array}$} \\
\hline & $\begin{array}{l}\text { Tambopata } \\
\text { (Sabaluyoc) }\end{array}$ & $\begin{array}{c}\text { Las Piedras } \\
\text { (Loreto) }\end{array}$ & $\begin{array}{l}\text { Laberinto } \\
\text { (Lagarto) }\end{array}$ & $\begin{array}{c}\text { Tahuamanu } \\
\text { (Alerta) }\end{array}$ & \\
\hline Área & 482,24 & 904,45 & 1592,65 & 975,79 & 988,78 \\
\hline Número de árboles & 169 & 872 & 410 & 667 & 529,50 \\
\hline Densidad (árboles/ha) & 0,35 & 0,96 & 0,26 & 0,68 & 0,54 \\
\hline $\begin{array}{l}\text { V1: Valor de producción } \\
\text { por árbol }(\mathrm{S} /)\end{array}$ & 78,55 & 63,18 & 68,03 & 59,93 & 67,42 \\
\hline $\begin{array}{l}\text { V2: Valor existencial o } \\
\text { per se por árbol }(\mathrm{S} /)\end{array}$ & 4295,45 & 5575,22 & 7706,42 & 2500,00 & 3941,44 \\
\hline $\begin{array}{l}\text { VT: Valor económico ecológi- } \\
\text { co por árbol (S/) }\end{array}$ & 4374,00 & 5638,41 & 7774,46 & 2559,93 & 4008,87 \\
\hline $\begin{array}{l}\text { VT: Valor económico ecológi- } \\
\text { co por concesión }(\mathrm{S} /)\end{array}$ & 739206,57 & 4916690,22 & 3187526,63 & 1707474,80 & 2122694,68 \\
\hline $\begin{array}{l}\text { VT: Valor económico ecológi- } \\
\text { co por hectárea concesión (S/) }\end{array}$ & 1532,86 & 5436,11 & 2001,40 & 1749,84 & 2146,78 \\
\hline
\end{tabular}

Cuadro 3. Valor económico ecológico del árbol de Bertholletia excelsa Bonpl. (castaña) en las cuatro concesiones

\begin{tabular}{lcc}
\hline \multicolumn{1}{c}{$\begin{array}{c}\text { Concesiones de } \\
\text { aprovechamiento de castaña }\end{array}$} & $\begin{array}{c}\text { Número total de } \\
\text { árboles por } \\
\text { concesión }\end{array}$ & $\begin{array}{c}\text { Valor económico } \\
\text { ecológico por } \\
\text { concesión S/ }\end{array}$ \\
\hline Tambopata (Sabaluyoc) & 169 & 739206,57 \\
Las Piedras (Loreto) & 872 & 4916690,22 \\
Laberinto (Lagarto) & 410 & 3187526,63 \\
Tahuamanu (Alerta) & 667 & 1707474,80 \\
\hline Media & 529,50 & $2637724,56 *$ \\
Desviación estándar & 305,75 & 626580,73 \\
Coeficiente de variación (\%) & 57,74 & 69,25 \\
\hline * Media aritmética del valor económico ecológico de las cuatro concesiones obtenida para \\
relacionar y determinar coeficiente de variación y correlación. \\
Nota: Nivel de confianza al 95\%.
\end{tabular}

Cuadro 4. Comparación del valor económico ecológico del total de árboles de Bertholletia excelsa Bonpl. (castaña) en las cuatro concesiones.

riores al encontrado en Brasil por de Sá et al. (2004) con S/. 1,59/kg. Estos resultados sirven como sustento para el presente estudio, debido a que los autores mencionados han empleado semejantes técnicas de extracción y mismos patrones para valorar.

Asimismo, el valor de los costos de producción por árbol, valor existencial o per se por árbol en pie, el valor económico ecológico del árbol, total de árboles y concesión o superficie, son diferentes en las cuatro concesiones en estudio. Esta variabilidad podría atribuirse a su estado fenotípico, genotípico, conservación y cultivo del árbol, así como también a su lejanía, accesibilidad, fisiografía y ubicación de la concesión (PROMAMAZONIA 2019, Gamarra 


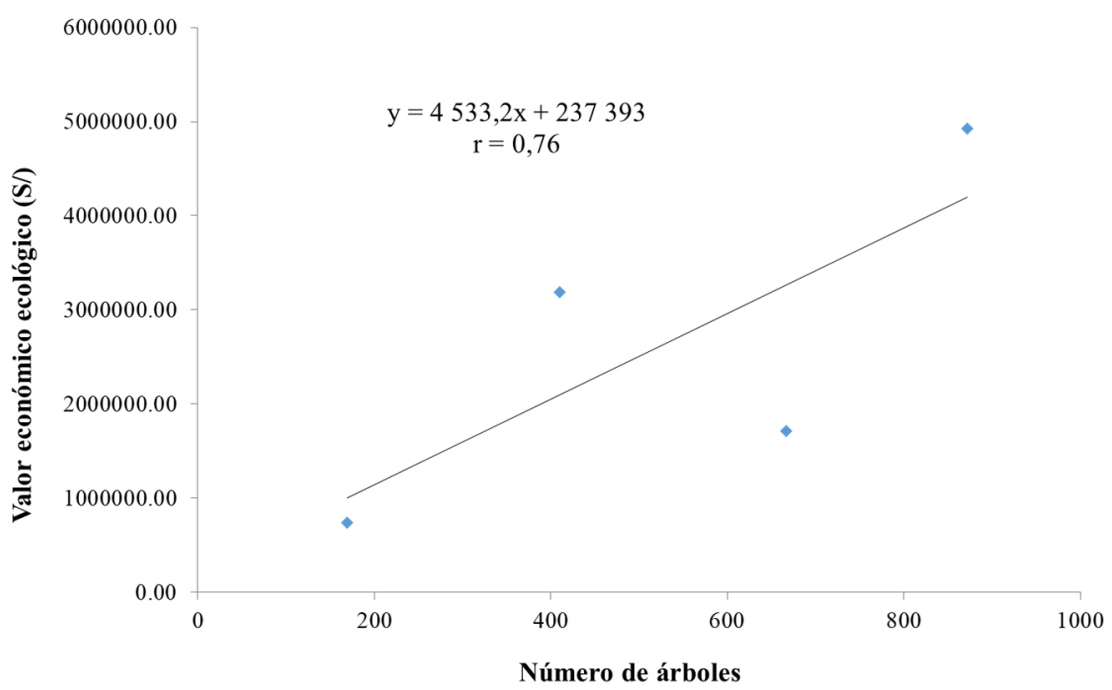

Figura 3. Relación de comparación del valor económico ecológico con el número total de árboles de Bertholletia excelsa Bonpl. (castaña) de las cuatro concesiones.

2014, Flores 1997). De ellos, los costos de los valores de producción de castaña en cáscara por árbol en los diferentes sectores son menores a lo encontrado por Rosales (2012b), quien reportó S/. 94,42/árbol, esto se debe a que dicho autor registró el valor de producción de frutos de castaña en forma pelada, el mismo que requiere de varios procesos de transformación más, por lo que el costo unitario seria mayor. Sin embargo, el valor existencial o per se por árbol en pie de la presente investigación (S/ 3 941,44) es muchísimo mayor comparado con lo reportado también por Rosales (2012b), que fue de S/. 422,40/árbol. Esto debido a que el mismo autor no considero la variable edad de los árboles en forma cuadrática, ni haber trabajado con el promedio de los diámetros, los mismos que reducen considerablemente los valores matemáticamente. Ello también habría afectado la valorización económica del árbol en sí de B. excelsa (S/. 516,82/árbol) (Rosales 2012b), así como los costos por accesibilidad y lejanía, razón por lo que se sustenta que el valor económico ecológico del presente estudio sería más de cinco veces mayor en los diferentes sectores.

\section{Conclusiones}

El valor de producción de la castaña en cáscara, el valor existencial o per se por árbol en pie y el valor económico ecológico del árbol de Bertholletia excelsa en las concesiones ubicadas en Tambopata, Las Piedras, Laberinto y Tahuamanu son diferentes, los mismos que estarían influidos por su estado de conservación, silvicultura, accesibilidad y ubicación de los árboles de castaña en cada concesión.

El valor económico ecológico por árbol de Bertholletia excelsa en la concesión ubicada en Laberinto (S/. 7 774,93) es mayor comparado a las ubicadas en Las Piedras (S/. 5 638,46), Tambopata (S/. 4 374,00) y Tahuamanu (S/. 2 559,93). Esto debido a que factores como la variable edad y diámetro de los árboles, e inaccesibilidad y lejanía de las concesiones aumentan el valor.

La relación del valor económico ecológico entre el número de árboles de cada concesión presenta una correlación significativa alta, el mismo que posibilita estimar una valorización económica ecológica por árbol de castaña, que para las cuatro concesiones evaluadas en la región de Madre de Dios seria de S/. 4 008,87. 
Valorización económica ecológica de árboles de Bertholletia excelsa

\section{Agradecimientos}

Al SERFOR CAF por el financiamiento económico e interés que tuvieron en la realización de esta investigación, a la Dirección Regional Forestal y Fauna Silvestre de Madre de Dios por la autorización respectiva y a los concesionarios castañeros de los diferentes distritos o sectores por brindar todo el apoyo incondicional e ingreso a sus áreas respectivas bajo manejo.

\section{Bibliografía}

Brienen, RJ. 2005. Tree rings in the tropics: a study on growth and ages of Bolivian rain forest trees. 1 ed. Riveralta, Beni, Bolivia. Promab scientific series 10. 144 p.

Collazos, JC. 2009. Manual de evaluación ambiental de proyectos. 1 ed. Lima, Perú. San marcos. 533 p.

Córdova, ZM. 1997. Estadística descriptiva e inferencial. 2 ed. Lima, Perú. Departamento de ciencias, facultad de ingeniería de la Pontificia Universidad Católica del Perú. 450 p.

de Sá, CP; Albano, MM; Correa, FA; Mota, DS; de Olivera, EL. 2004. Custo e rentabilidade para o sistema melhorado de extracao de castanha-do-brasil na reserva extrativista Chico Mendes no Acre. Embrapa. Rio Branco, Brasil. Comunicado técnico ${ }^{\circ} 162.4$ p. Consultado 20 jul. 2019. Disponible en https://ainfo.cnptia.embrapa.br/digital/bitstream/CPAFAC-2010/21171/1/comunicado170.pdf

DRFFS - MDD (Dirección Regional Forestal de Flora y Fauna Silvestre - Madre de Dios). 2019. Balance de extracción de castaña en cascara. Puerto Maldonado, Madre de Dios. CIEF-MDD. 9 p.

Flores, SP. 1997. Cultivo de frutales nativos amazónicos: Manual para el extensionista. Tratado de cooperación amazónica. Lima, Perú. Secretaria Pro Tempore - IIAP. 307 p.

Gamarra, EJ. 2014. Costos generados a lo largo de la cadena de la castaña amazónica: Asistencia técnica del apoyo presupuestario a la políti- ca de promoción de productos ecológicos. Madre de Dios, Perú. EURO ECO TRADE. 31 p.

ICONA (Instituto para la Conservación de la Naturaleza). 1975. Manual de valoración de pérdidas por incendios forestales. Ministerio de agricultura, pesca y alimentación. Ed. ICONA. Madrid. 115 p.

PROMAMAZONIA (Centro de Promoción de la Biodiversidad Amazónica). 2019. Castaña orgánica, descripción de la cadena productiva y flujo del producto: La cadena de valor de la castaña amazónica. 4 p. Consultado 20 jul. 2019. Disponible en http://www.promamazonia.org.pe/SiBiocomercio/wfr LineaProductivaItem.aspx?Tef IdTemaFicha $=163$

Rosales, ES. 2012a. Plan de manejo forestal de productos diferentes de la madera (Bertholletia excelsa). Edsfor E.I.R.L. Puerto Maldonado, Madre de Dios. 67 p.

Rosales, ES. 2012b. Valoración económica de árboles de castaña (Bertholletia excelsa HBK) en una concesión castañera de 3 155,06 has fundo Santa Julia, Las Piedras, Tambopata - Madre de Dios. Lima. Trabajo técnico, EPG-UNFV. 56 p.

SERFOR CAF (Servicio Nacional Forestal y de Fauna Silvestre para la Corporación Andina de Fomento). 2018. Evaluación de cuatro concesiones castañeras para su valorización económica ecológica de árboles de Bertholletia excelsa Bonpl. por el método producción-daños en Madre de Dios. Metodología de investigación. Puerto Maldonado, Madre de Dios. 7 p. Informe $\mathrm{n}^{\circ} 01$.

SERFOR CAF (Servicio Nacional Forestal y de Fauna Silvestre para la Corporación Andina de Fomento). 2019a. Evaluación de cuatro concesiones castañeras para su valorización económica ecológica de árboles de Bertholletia excelsa Bonpl. por el método producción-daños en Madre de Dios. Puerto Maldonado, Madre de Dios. 100 p. Informe $\mathrm{n}^{\circ} 02$.

SERFOR CAF (Servicio Nacional Forestal y de Fauna Silvestre para la Corporación Andina de Fomento). 2019b. Evaluación de cuatro 
concesiones castañeras para su valorización económica ecológica de árboles de Bertholletia excelsa Bonpl. por el método producción-daños en Madre de Dios. Puerto Maldonado, Madre de Dios. 22 p. Informe $n^{\circ} 03$. 